\title{
3D MODELING AND DATA ENRICHMENT IN DIGITAL RECONSTRUCTION OF ARCHITECTURAL HERITAGE
}

\author{
F.I. Apollonio ${ }^{\text {a }}$, M. Gaiani ${ }^{\text {a }}$, Z. Sun ${ }^{\text {a }}$ \\ ${ }^{a}$ Dipartimento di Architettura - Alma Mater Studiorum Università di Bologna, Bologna, Italy - (fabrizio.apollonio, \\ marco.gaiani, zhengsun2)@unibo.it
}

KEY WORDS: Knowledge system, Architectural heritage, Semantic structure, 3D modeling from drawings, Real time rendering, Uncertainty visualization.

\begin{abstract}
:
The paper presents some experiments carried out as part of the virtual reconstruction of buildings just documented by partial sketches, or partially built, or no more existing, with the aim (a) to emphasize the use of a semantic construction of the digital model, not only as a means to modeling a building but as a cognitive system, (b) to show conceptual similarity between the treaties and $\mathrm{BIM}$, (c) to propose new and more robust solutions to the 3D modeling from $2 \mathrm{D}$ drawings for $\mathrm{CH}$ artifacts, able to allow the verification of the assumptions used during the reconstruction pipeline, (d) to make use of interactive technical reference, typically real-time photorealistic rendering, for the visualization of three-dimensional model and of variants snapshots, managed by an iconic for illustrating the method of comparison and guided reading of model's characters of the steps taken.
\end{abstract}

\section{INTRODUCTION}

The today development of new and more effective digital technologies, such as knowledge/building information systems, real-time rendering of $3 \mathrm{D}$ models, multimedia techniques, animations and simulations has opened new scenarios for reading and interpreting Architectural Heritage (AH), where all the information become available in a visual and integrated way. Digital systems introduce the possibility of interchangeable media able to offer multiple access to a given term or object, and enable a multidimensional approach to knowledge on several levels. 3D architectural models have become 'spatial metaphors', enabling the distribution of pieces of information in time and space (Blaise \& Dudek, 2007) in order to be used as interface for the localization and retrieving data associated. Summarizing, 3D model-based structured systems allows to activate, types of investigation previously inconceivable in specific task of the $\mathrm{AH}$ field: archiving and accessing data, spatial analysis, simulation of un-built projects, etc.

Architecture, in fact, can be understood as a cognitive system in which rules for its composition (e.g. symmetry, arrays, proportions) and construction are largely defined (Tzonis \& Lefaivre, 1986). The most famous architecture book of ever, the Andrea Palladio's I Quattro libri dell'architettura (Venezia, 1570), i.e., is organized as a knowledge system. In this treatise Palladio explains his compositional and constructive system of architecture as a knowledge parameterized system underling a standardized procedure, from the whole edifice to its detail, and a limited set of rules to move from the design to the construction on-site.

Being all $\mathrm{AH}$, so more or less, based on organizational systems such as those of the architect of Rotonda, it is as clear that an excellent solution to fit this system is the use of $3 \mathrm{D}$ digital semantic models organized as cognitive systems with geoobject items in a 3D Information System (Manferdini et al., 2008).

The availability of $3 \mathrm{D}$ semantic models organized as cognitive systems allows to have a semantic approach to the classical problem of building design, management and understanding.

A knowledge-based architectural system may propose, i.e., new meanings of the concept of architectural representation, adding an extra dimension, temporal one (diachronic and synchronic), which allows to know not only the evolution and transformation during life cycle of an architectural artifact, but also the exhaustive documentary base covering the whole process of research related to the creation of digital content within the reconstruction pipeline.

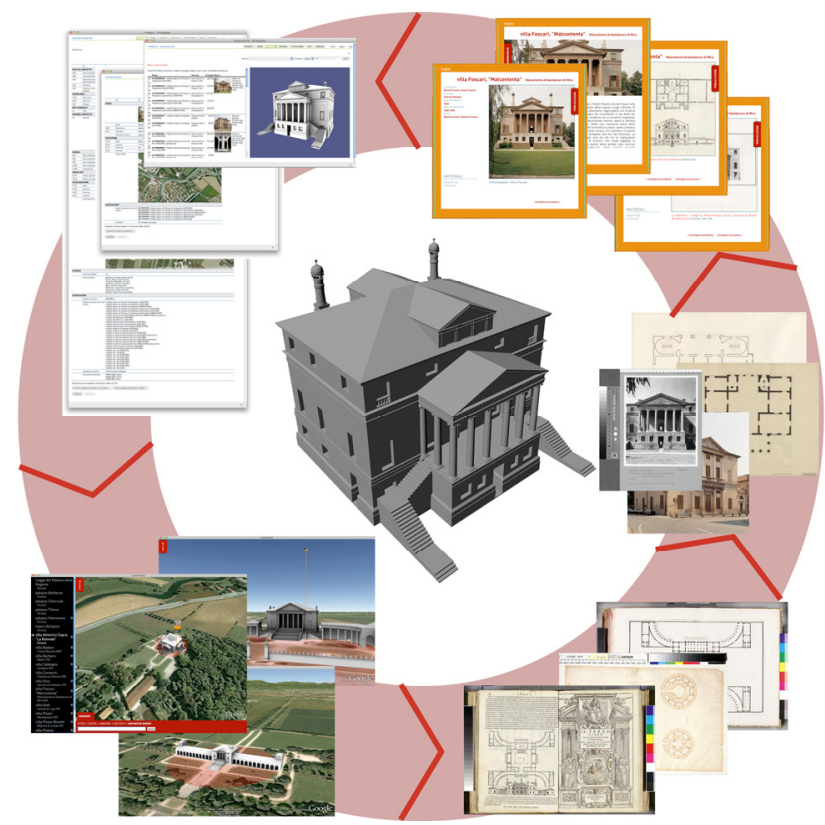

Figure 1. Data relationship structure and 3D model as browsable interface

In this paper we presents some improvements to the simple reconstruction of $\mathrm{AH}$ no longer existing or un-built or missing stages by means of 3D models. Our work, developed entirely using commercial software and a limited number of scripts, aim to develop a complete pipeline to have final 3D models able to show using high quality RTR rendering and semantic structures not only the as-built or the original state, or some intermediate stages, but the design intentions and the construction constraints and variants. 


\section{AH 3D MODELING AND HISTORICAL RECONSTRUCTION}

Wide is the series of reconstructions works of design hypotheses and drawings that, as early as 1990 (ENSAM - IBM, 1992) have used 3D digital modeling techniques. Koller et al., (2009) in the resettlement of the general framework of the challenges and opportunities offered by 3D models digital archives related to cultural heritage, recognized the need to make visible the traceability of all additions, subtractions, and changes to $3 \mathrm{D}$ models, in order to let understandable the calculation and display of differences between 3D models of the object/artifact. Gabellone (2012), following on the principle 7 of the Charter of Seville, has focused attention on the full transparency of methods, techniques and documentation in supporting a project of virtual reconstruction (in archeology), the only tool able to validate the results of any reconstructive study and guarantee future generations a review of the results without needing to restart the job from the beginning.

Within this context, a huge amount of studies have resulted in order to define new protocols for processing (acquisition, manipulation and management) spatial data, resulting in the development of new methodologies for the study and openness to further research scenarios.

An important contribution in this field has come from Blaise e Dudek who introduced, in example, the genesis of an informative modeling methodology (2005), in which the representation of architectural objects is used for information search and visualization, letting them able to define some methodological approach in order to increase la intelligibility of 3D models' informative contents. Aiming to improving the comprehension of the complex and discontinuous process of knowledge acquisition, Same authors (2007) also introduced a generic formalism of information integration that lets the researchers to gather indications step by step, and allows them to visually follow up the knowledge acquisition process.

De Luca et al. described a methodological approach to let usable existing iconographic 'corpus' for the analysis and the 3D management of building transformations. The aim was to establish a relation between the iconography used for the hypothetical reconstruction and the $3 \mathrm{D}$ representation that depends on it. This approach relates to the idea of using 3D representations like visualization systems capable of reflecting the amount of knowledge developed by the study of a historic building (2010). In a recent paper, De Luca and Lo Buglio addressed the issue of the review of the methodological aspects concerning the collection of information that describes an architectural object in order to measure the benefit in terms of information provided by the document, with reference to the requirements and the means employed (2012), offering an approach to the creation of representation systems that articulate the digital instance with the geometric/semantic model.

Recent work of our group included within the series of fruitful experiences of reconstruction of an entire project from partial sketches (Apollonio et al., 2011), puts some significant innovations with regard to:

1. the verification of the hypothesis of drawing to be the original project for the first nucleus of a palladian villa, during the century widely reshaped (Beltramini, 2010);

2. the use of a semantic construction of the digital model, not only as a means to look for a building such a cognitive system;

3. the use of interactive technical reference - typically realtime photorealistic rendering for the visualization of three-dimensional model and the use of variants snapshots managed by an iconic for illustration using the method of comparison and guided reading of model's characters of the steps taken.

Through the semantic structure of digital models and the use of Real Time Rendering (RTR) techniques we attempted to develop a process of acquiring knowledge able to point out and let understandable, as well as reusable, the analysis of preliminary data and interpretation criteria used, in order to validate the whole process, giving the ability to visually assess our level of knowledge, with its flaws and lacunae, and to carry out comparative operations on the set of data and information held, allowing the compatibility of the digital model with alternative techniques of representation.

\section{TREATISES AND BIM AS KNOWLEDGE SYSTEM}

A powerful structured development of the concept of semantic 3D modeling is the Building Information Modeling (BIM), which is able to define objects parametrically.

BIM is a building design and documentation methodology characterized by the creation and use of coordinated, internally consistent computable information about a building project in design and construction. From these characterizations is possible to reveal that classical building composition and construction and parametric BIM are closely linked, and BIM will be an excellent technique to build knowledge-based architectural system.

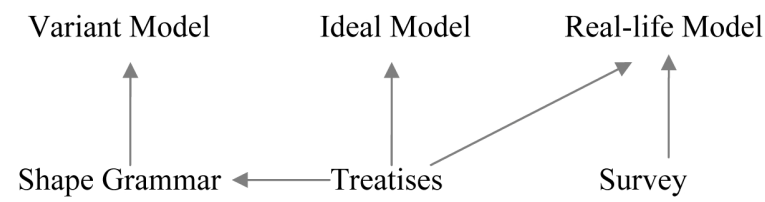

Figure 2. Central role of treatises in producing different models

The transition from CAD to BIM (Building Information Modeling) in AEC (Architecture, Engineering and Construction) industry is already underway (Eastman, 2009), while the documentation and management of $\mathrm{AH}$ has barely benefited from this technological renovation (Gaiani, 1999). The applications of BIM in new projects remind us that BIM is not only a powerful modeling tool, but also provides inherent semantic data pertaining to structural, material and operational information (Eastman et al., 2007). Therefore BIM is expected to be a central database supporting comprehensive data input for life-cycle management, and where multiple data are collected, stored and retrieved for various purposes. Besides explicit semantic description, BIM also facilitates the theoretical and historical study via historical documents enrichment (Pauwels et al., 2008) (Fai et al., 2011). Current BIM platforms are capable of object-based parametric modeling (Aish \& Woodbury, 2005). Instead of drawing concretes shapes, users first define the category (window, door, column, etc.) of the objects, and then establish the parametric frameworks. Objects of different categories vary in semantic meanings and inherent behaviors. The parametric geometry allows accurate and flexible generation of various dimensions and automatic adaptation to other components. These attributes are closely analogous to classical architecture's proportional semantic structure. Because of this close link between classical building composition and construction and parametric BIM, it would be an excellent tool to build knowledge-based architectural system. The BIM models are expected, in fact, to ease the modeling process and to provide comprehensive data shared among different platforms for further simulations, even if, among these researches' current field of architectural heritage approaches, several common problems are to be addressed, such as (a) the 
integration of BIM platforms and data capturing technologies for existing buildings, (b) standard of data exchange in BIM as well as BIM and other platforms, (c) multiple data enrichment to the geometric model.

Analogy between BIM and Renaissance architectural treatises could be built if we regard both of them as knowledge systems. In the light of data collection, users of BIM confront similar problems as authors of treatises. Just as Palladio and other architects or theorists built their written works partly on the precedents and partly on their own observations of antique ruins, people modeling a historical building or a piece of component have to determine the origins of data with respect to different motivations. In spite of improvement of data capturing technologies, knowledge from treatises is still a neglected reference in modeling and documentation of architectural heritage (Figure 2).

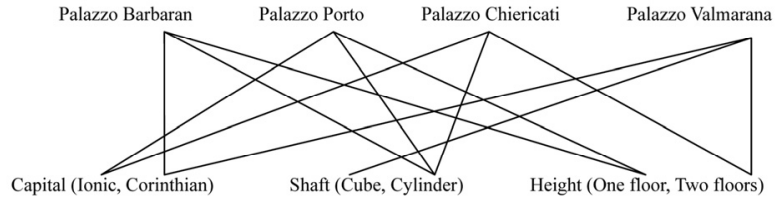

Figure 3. Options of rule 1 applied to Palazzo Barbaran, Palazzo Porto, Palazzo Chiericati and Palazzo Valmarana

Among the knowledge-system structured treatises, Palladio's $I$ Quattro libri dell'Architettura is a typical example (Baldissini et al., 2008). He sets up the grammar of geometric composition from single components to building types, and demonstrated great flexibility via the exhibition of Palladio's works. Therefore, it is feasible to employ the plans, elevations and details in the book to build accurate 3D models of Palladio's architecture. Four Books of Architecture present two important properties for computer applications: they show the Palladian buildings theoretical foundation as complete knowledge system, and they demonstrate a rigid formalism that well fits computer features and techniques (Wittkover, 1962). For these reasons many methods and techniques based on Palladian grammars, mainly generative techniques such as Palladian villa production with 3D printer (Sass, 2007) and generative algorithm applied to Villa Rotonda (Park, 2007), were developed during the last 30 years.

\subsection{From column to Palace: a bottom-up process}

Architectural treatises provide large amount of historical data including dimensions and shapes. BIM-based modeling extracts knowledge from these data and draws reference from the way data is collected and represented, as in the case of parallel of orders.

Extracting the semantic organization and modular dimensions from treatises of Palladio, Scamozzi and Vignola, we modeled the parametric Doric orders using Autodesk Revit Architecture 2012. The workflow consists of:

1) creating a set $2 \mathrm{D}$ constraints labeled with parameters according to semantic nodes;

2) setting up the parameters constraints by formulas;

3) creating geometry of the semantic nodes and aligning them to the corresponding reference planes;

4) loading the profile to the column family and sweeping it along the bottom edge of the order.

5) creating another profile and loading it into the column family to generate the fluted order via Boolean operation.

Using this workflow we are able to generate a set of types in a wide range of dimensions by assigning new values to the 'module' parameter. The ideal models strictly based on treatises enable automatic generation of knowledge-based components and serves as central platforms to which data captured from surveys could be linked. The parallel of Doric orders in BIM platform provides a set of views similar to the parallel of orders in the treatises, and also creates schedules of the data in a parallel way. New forms could be produced by modifying parameters of profiles or substituting profiles. If we take into account of the paths along which profiles are swept, the possibilities of formal compositions are multiplied. I.e. in Palazzo Barbaran da Porto, one of most beautiful Palladio's palaces, the triangular cornice and curve cornice above the windows on the façade in noble floor share the same profile but different path, and the cornices of noble floor and 3rd floor have different profiles but the same path (Figure 4, 5).

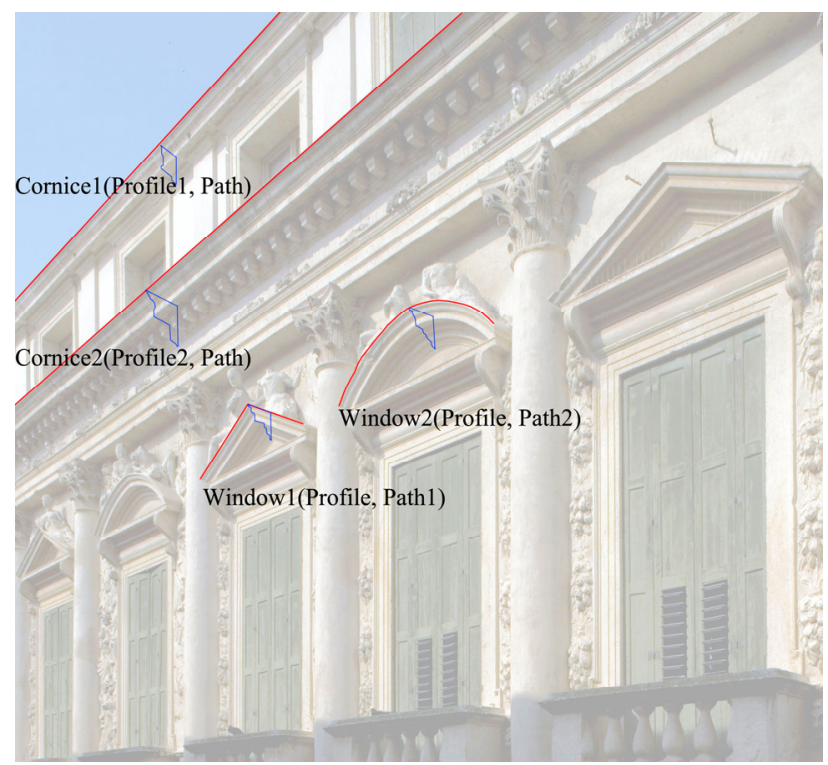

Figure 4. Two groups of components sharing the same path and profile in Palazzo Barbaran da Porto

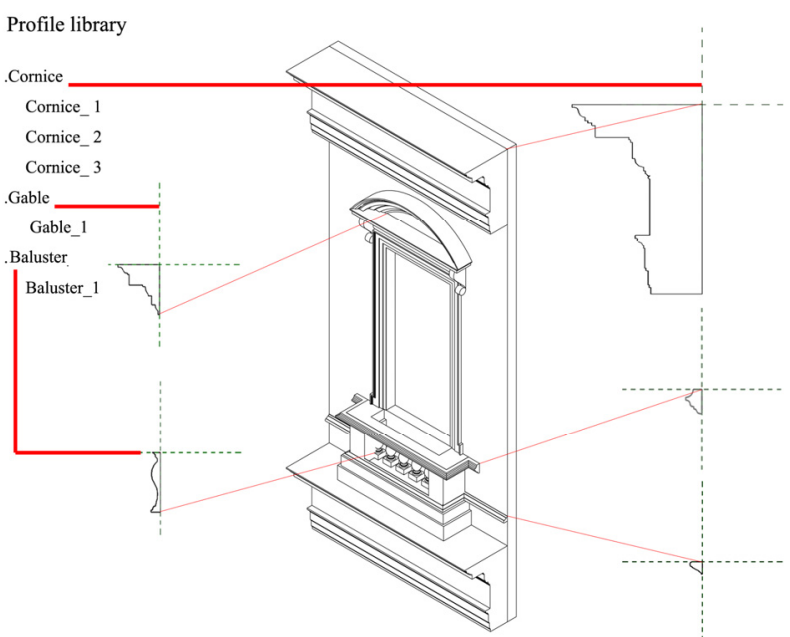

Figure 5. A unit of facade of Palazzo Barbaran da Porto and its profile library

The BIM-based modeling approach can be applied to establish a library of architectural elements catalogued semantically (Apollonio et al., 2012). New geometry in a specific category could be generated via profile edition strictly based on grammar, and we can extend our scope to a set of components that are semantically organized, such as in Palazzo Barbaran da Porto. 
A basic unit that composes the façade of Palladio's Palazzo (Figure 6) is usually articulated with four rules:

1. a pair of pilasters whose capital could be either Ionic or Corinthian, shaft either cube or cylinder, height dominating either one floor or two floors;

2. a window with top molding's cornice either triangular or curve (if the pilasters dominate two floors in rule one, then a rectangular window is added either above or below the window on the noble plan);

3. a balcony with cornice and an array of balusters;

4. two segments of entablatures on the bottom and top of the façade unit.

We realize the Bottom-Up process from semantic nodes to the whole façade, keeping in mind that the options listed in each of the four rules are in essence parameters of dimensions, visibility and substitution. The key idea was to use the link between Revit Architecture and the external database used to create the constraints (i.e. Microsoft Access, Microsoft Excel, or ODBC database), modifying the values of parameters. As metadata of model are catalogued semantically with optional parameters in the database, it provides user with interfaces to make modification of dimensional parameters (values with number) and Boolean (values: yes or no) parameters. In this way, the Bottom-Up process could be realized via data modification in Access.

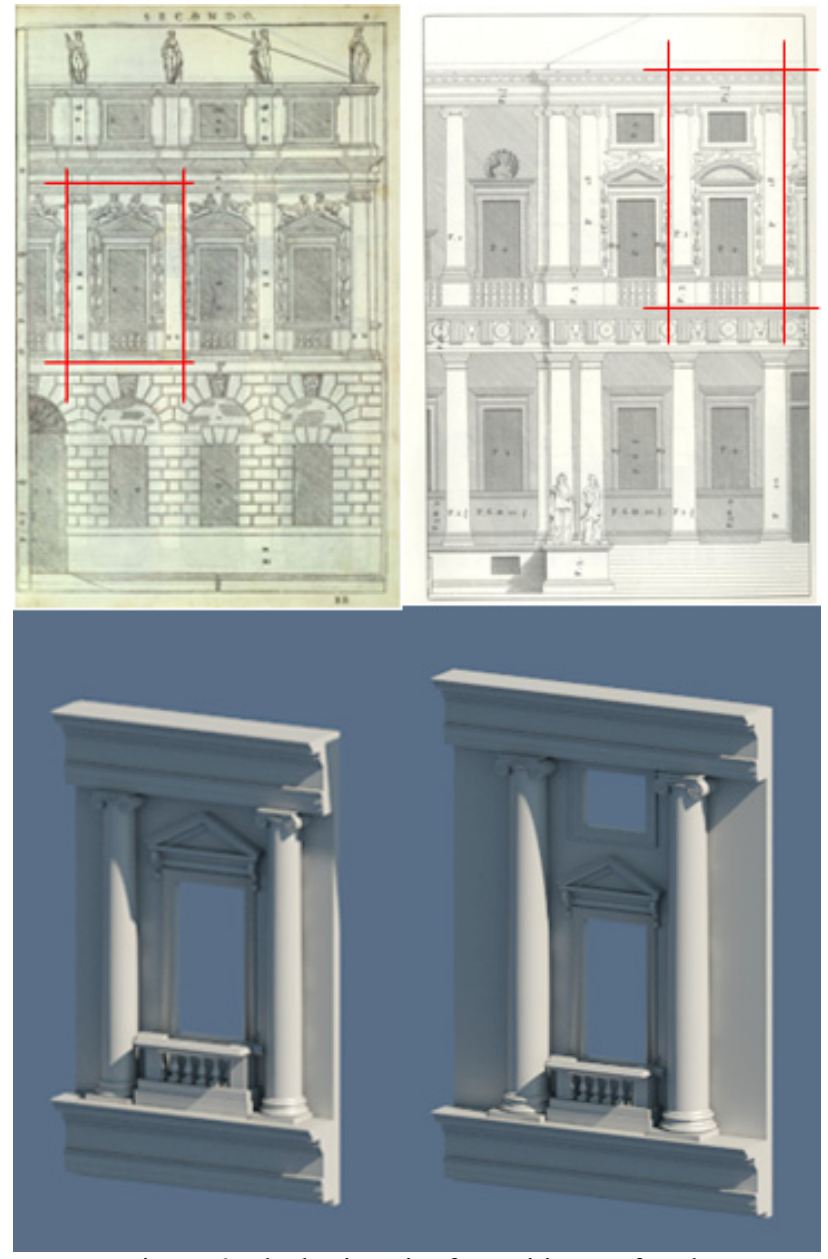

Figure 6 . The basic unit of repetition on façade

\subsection{Data enrichment and displaying the uncertainty}

The parametric models established geometric frameworks, to which multiple data in a wide range of domains, such as structure, material and real life state could be assigned. Parameters are the central attributes of BIM platforms. They could be shared in the level of category, family and type, or not shared as instance attributes.

Category represents the semantic domain of objects; family refers to models with unique parametric frameworks in the category; a family consists of a set of types with different dimensions; and instance means a concrete piece of object with unique ID in a project.

In the family level, the parameters not only include geometric dimensions. For example, we can assign visibility parameters with the value 'yes/no' to the fluted void of Doric columns. As a result, when the value is 'yes', the Doric order is fluted, and vice versa when the value is 'no' the column is smooth. In the same way we could produce a set of variants evolved from the original type and save them as different types.

To move from design to as-built models, data captured from surveys could be added in the type level and instance level. When high geometric accuracy is required, photogrammetry and laser scanner could be employed to generate precise mesh surfaces linking with the BIM model.

Within this framework it is possible to use BIM-based 3D AH models in order to qualify and authenticate products of scientific research, too linking specific parts of the models with the corresponding text metadata (catalogue, commentary and bibliography) that allows transparency of information to users, and to become demonstrative of the solutions adopted to meet the uncertainties and the lacks. Many of 3D architectural models are, in fact, reconstruction of buildings just designed, no longer existing, nor fully documented; therefore, it is inevitable that such reconstructions contain a small or large amount of hypothetical elements, characterized by their specific degree of uncertainty. This is due to a set of factors:

(a) the need to make up for the missing parts in the today structure;

(b) the need to implement hypothesis in the reconstruction of a 2D drawing in a spatial shape,

(c) the temporization of the various layers in order to decide an original state;

(d) the different conformations in time.

Beyond the analysis specifically conducted (ex-post), digital systems allow the collection and systematization (ex-ante) of operations in order to determine the geometry of the elements that contribute to the definition of an architectural work, becoming themselves explanatory values of the geometricformal genesis of that building. The possibility to examine the collection of data containing large amounts of records, such as geometric analysis and semantic characterizations of artifacts, offer new approaches to the classification and the comparison.

It is vital, therefore, that informative/cognitive systems facilitate the evaluation of the documents relating to the uncertainties inherent in models reconstructed, and which may be related to:

- $\quad$ architectural / structural elements;

- $\quad$ size / geometry;

- $\quad$ stylistic / formal;

- temporal correspondence;

- building materials.

Such type of information may be conveyed through

(a) a new 3D symbology (e.g. a series of glyphs 3D);

(b) animation techniques display;

(c) rendering techniques;

(d) a combination of text metadata and $3 \mathrm{D}$ visualization.

We preferred to give solution to uncertainty display defining a structured modeling process, based on different levels of interpretation recalling that BIM's semantic structure enables multiple data enrichment and filter according to various analysis need. Also, multiple data could be enriched in different levels of 
category, family, type and instance out of different motivations. It gave access to potential simulation by sharing data among various platforms.

We defined 8 level of reconstruction process characterized by a progressively increasing uncertainty:

1. reconstruction based on original design or survey drawing;

2. reconstruction based on sketch, therefore affected by a low level of dimensional accuracy;

3. reconstruction based on design reference dated to the same period of the drawing or building studied and which have significant stylistic similarities (e.g., coverage, type of roof, gutter frame, frames, roof, basement, or the openings and decorative system) with that drawing/building;

4. reconstruction based on reference to treaties, books or architectural guideline written by the architect, author of drawing or project studied;

5. reconstruction based on reference to treaties or manual to which the architect has or could have used as his own reference (e.g. measurements of the rooms, stair design, detail design and equipment, the architectural orders, if any, as well as for the definition of the height of the internal doors or types and sets the height of the time);

6. reconstruction based on interpretative hypotheses referring to specific architectural style;

7. reconstruction based on interpretative hypotheses more thrusts, referring to the construction systems at that time in order to achieve solutions constructively plausible and compatible with the project, by which, however, is not always possible to reach conjecture or univocal solutions;

8. reconstruction based on reconstructive conjectures failing references.

Reconstruction based on the uncertainty display uses a density slicing color code - a variation of pseudo color - that divides the rendering objects into a few color bands, corresponding to each level of interpretation/uncertainty (Figure 7).

For each level we then defined a level of modeling in Revit with specific constraints and the need to compile suitable metadata.

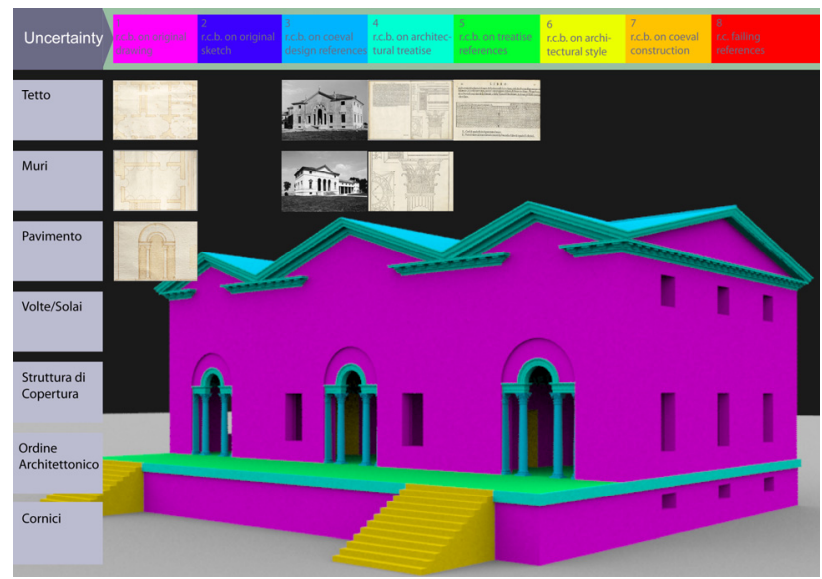

Figure 7. A. Palladio, Villa for a twins. 3D modeling reconstructive conjecture uncertainty map

\section{BEYOND THE SEMANTIC STRUCTURE OF INFORMATION: THE COGNITIVE GRAPH}

Systematizing geometric information with uncertainty related needs to take into account heterogeneous information, able to considering the evolution of our knowledge, producing 2D/3D dynamic graphics, and adapting our practices to the specific manners of the AH field.
Studying and displaying evolution of an artifact (or ensemble of artifacts) requires introduction of temporal and documentary additional dimensions.

Temporal dimension allows to distribute pieces of information in space and in time: in a certain position; at that time of the study; at that moment of the history.

Documentary one allows to display, or let understandable and evaluable, the methodology used in analyzing the architectural evolutions of that artifact, based on interpretation and comparison of various types of documentation.

3D model representation, as metaphor of cognitive system related to architectural corpus do not show us the 'real' object, but how we understand it: different pieces of documentation in relation to architectural elements could become a browsing tool, allowing to mediate between the information to be handled and users able to investigate sets of data or information element by element.

The result is a cognitive graph, as visual metaphor of case study, which aims to restore the hierarchical structure that governs the geometric definition of the 3D model and gives access to documents about that studied artifact.

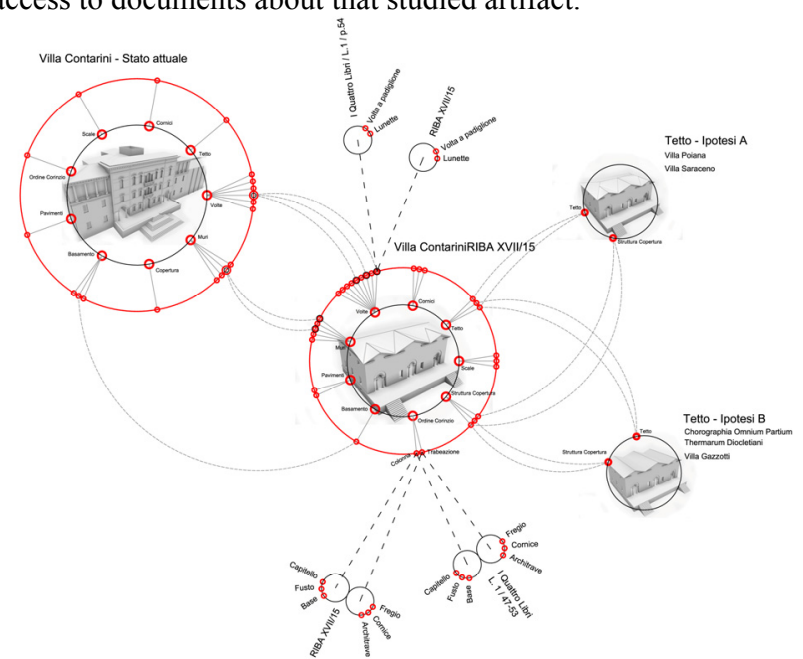

Figure 8 . The cognitive graph

Due to the relationship between each document in a data set and its corresponding element of the artifact (building as a whole, parts, details, etc.) information structure could be visualized and retrieved inside a 3D model/interface that combines the metaphoric figure of cognitive system. Through this structure, exploiting the semantic graphic codes, representation is able to:

- underline inconsistency in the documentation or its analysis;

show levels of incompleteness of the investigation;

- provide an updated visualization of our knowledge on an object.

We implemented the cognitive graph using high quality RTR visualization techniques with the aim to obtain fully 3D interactive comparison and interpretation of different solutions and states guided by the $3 \mathrm{D}$ model features and construction steps. Our RTR models are browsable according to three different reading levels, always retrievable, that allows:

1. comparison between existing drawing and its 3D model;

2. analysis of the geometric characteristics of the model, through the use of patterns and diagrams that facilitate the reading of correspondence and/or anomalies identified during the analysis of the drawing, or the criteria of ratio used to design its parts;

3. photorealistic rendering of reconstructed project within its hypothetical/original context, as well as matching any hypotheses alternative to the shaping of some of its parts. 


\section{CONCLUSIONS}

The solution adopted allows many types of analysis and could introduce new and meaningful innovations to the architecture interpretation methods and techniques, along two different paths.

The first involves proposing photorealistic images obtained using simulated global illumination rendering techniques. It is, obviously, a further development of Renaissance representation advancements and a new step of the desire of fifteenth and sixteenth-century artists and intellectuals to use drawing to investigate the world and the nature.

The second concerns a truly interpretative work, obtained from the simplification operation, inherent the schematism embedded in the concept of 'model'. This simplification allows creating visualizations of the real more interpretative tan philological or perceptually correct. In this case reconstruction means a desire to explicitly describe and represent a whole story: not only a 're-drawing' to understand but a 're-interpretation' to communicate.

\section{REFERENCES}

Aish, R., \& Woodbury, R., 2005. Multi-level Interaction in Parametric Design. In: SG 2005 Conference Proceedings: International Symposium on Smart Graphic, Frauenwörth Cloister, Germany, pp. 151-162.

Apollonio, I. F., Gaiani, M., Beltramini, G., Fabbi, G., 2011. Villa Contarini a Piazzola sul Brenta: studi per un'ipotesi di attribuzione palladiana servendosi di modelli tridimensionali. Disegnare idee immagini, 42, pp. 42-55.

Apollonio, I. F., Gaiani, M., Sun Z., 2012. BIM-based modeling and data enrichment of classical architectural buildings. SCIRES-IT, 2(2), pp. 41-62.

Baldissini, S., Beltramini, G., Gaiani, M., 2008. Rich internet application as paradigm to manage and visualize cultural heritage references and contents: the case study of the first electronic edition of Andrea Palladio's The Four Books of Architecture. In: DMACH conference proceedings, Amman, Jordan, pp. 255-270.

Beltramini, G., 2010. Andrea Palladio. Plan and elevation of a villa for two brothers (Villa Contrarini at Piazzola?). In: Murray, I., Charles, H., 2010, Palladio and His Legacy, A transatlantic Journey. Marsilio, Venezia, pp. 75-76.

Dudek, I., Blaise, J. Y., 2005. From Artefact Representation to Information Visualisation: Genesis of Informative Modelling. In: BUTZ, A., et al. (Eds.): 8th Smart Graphics International Conference. Springer-Verlag, Berlin Heidelberg, p. 230-236.

Blaise, J. Y., Dudek, I., 2007. Visual tools decipher historic artefacts documentation. In: Journal of Universal Computer Science. 7th International Conference on Knowledge Management, Salamanca, Spain, pp. 456-463.

De Luca, L., Busarayat, C., Stefani, C., Renaudin, N., Florenzano, M., Véron, P., 2010. An Iconography-Based Modeling Approach for the Spatio-Temporal Analysis of Architectural Heritage. In: Shape Modeling International Conference (SMI '10). Washington, USA, pp. 78-89.

Dudek, I., Blaise, J. Y., 2005. From Artefact Representation to Information Visualisation: Genesis of Informative Modelling. In
Butz, A., et al. (Eds.): 8th Smart Graphics International Conference. Berlin Heidelberg, Springer-Verlag. pp. 230-236.

Dudek, I., Blaise, J. Y., 2005. Using Abstraction Levels in the Visual Exploitation of a Knowledge Acquisition Process. In: $i$ KNOW' 05 Proceedings of the 5th International Conference on Knowledge Management and Knowledge Technologies. Graz, Austria, pp. 543-552.

Eastman, C., 2009. "What is BIM?", http://bim.arch.gatech.edu/?id=402 (21 june 2013)

Eastman, C., Teicholz, P., Sacks, R., Liston, K., 2007. BIM Handbook: A Guide to Building Information Modeling for Owners, Managers, Designers, Engineers and Contractors. John Wiley \& Sons, New Jersey.

ENSAM - IBM, Mémoires de pierre, 1992, video.

Fai, S., Graham, K., Duckworth, T., Wood N., Attar R., 2011. Building Information Modeling and Heritage Documentation. In: CIPA 2011 Conference Proceedings: XXIIIrd International CIPA Symposiu. Prague, Czech.

Gabellone, F., 2012. La trasparenza scientifica in archeologia virtuale: una lettura critica al principio n.7 della Carta di Siviglia. SCIRES-IT, 2(2), pp. 99-124.

Gaiani, M., 1999. Translating the architecture of the world into virtual reality and vice- versa: 7 years of experimentation with "conservation and representation" at OFF. In: Proceedings of Heritage Applications of $3 D$ Digital Imaging, Ottawa, Canada.

Koller, D., Frischer, B., Humphreys, G., 2009 Research Challenges for Digital Archives of 3D Cultural Heritage Models. Journal on Computing and Cultural Heritage, 2(3).

Lo Buglio, D., De Luca, L., 2012. Representation of architectural artifacts: definition of an approach combining the complexity of the 3D digital instance with the intelligibility of the theoretical model. SCIRES-IT, 2(2), pp. 63-76.

Manferdini, A.M., Remondino, F., Baldissini, S., Gaiani, M., Benedetti, B., 2008. 3D Modeling and Semantic Classification of Archaeological Finds for Management and Visualization in3D Archaeological Databases. In: Proceedings of the 14th International Conference on Virtual Systems and Multimedia, Cyprus, pp. 221-228.

Park, H. J., 2007. Parametric variations of Palladio's Villa Rotonda. International Journal of Architectural Computing, 6(2), pp. 145-169.

Pauwels P., Verstaeten, R., De Meyer, R., Van Campenhout, J., 2008. Architectural information modelling for virtual heritage application. In: Proceedings of the 14th International Conference on Virtual Systems and Multimedia, Cyprus, pp. 1823 .

Sass, L., 2007. A Palladian construction grammar-design reasoning with shape grammars and rapid prototyping. Environment and Planning B: Planning and Design, 34(1), pp. 87-106.

Tzonis, A., Lefaivre L., 1986. Classical Architecture, The Poetics of Order. MIT Press, Cambridge.

Wittkover, R., 1962. Architectural Principles in the Age of Humanism. Academy Editions, London. 